\title{
Drawing Planar Graphs with Circular Arcs
}

\author{
C. C. Cheng ${ }^{\star}$, C. A. Duncan ${ }^{\star \star \star \star \star}$, M. T. Goodrich ${ }^{\star \star \star}$, and S. G. Kobourov ${ }^{\star \star \star}$ \\ The Johns Hopkins University \\ Baltimore, MD 21218
}

\begin{abstract}
In this paper we address the problem of drawing planar graphs with circular arcs while maintaining good angular resolution and small drawing area. We present a lower bound on the area of drawings in which edges are drawn using exactly one circular arc. We also give an algorithm for drawing $n$-vertex planar graphs such that the edges are sequences of two continuous circular arcs. The algorithm runs in $O(n)$ time and embeds the graph on the $O(n) \times O(n)$ grid, while maintaining $\Theta(1 / d(v))$ angular resolution, where $d(v)$ is the degree of vertex $v$. Since in this case we use circular arcs of infinite radius, this is also the first algorithm to simultaneously achieve good angular resolution, small area and at most one bend per edge using straight-line segments. Finally, we show how to create drawings in which edges are smooth $C^{1}$-continuous curves, represented by a sequence of at most three circular arcs.
\end{abstract}

\section{Introduction}

The study of methods for rendering planar graphs is central in the graph drawing literature. In planar graph drawings, vertices are represented by distinct points in the plane and edges are drawn as continuous curves that do not cross one another [1]. An important characteristic of a graph drawing is its readability, and some of the essential qualities that determine readability include the following:

1. edge smoothness: edges should be drawn with "smooth" curves. Ideally, we prefer straight line segments. If some other considerations prevent the use of straight lines, then edges should be drawn as simple smooth low-degree curves or polylines with few bends.

2. area: vertices and bend points should be placed at integer grid points in as small a box as possible. Ideally, vertices and bend points should be placed on an $O(n) \times O(n)$ grid, where $n$ is the number of vertices in the graph.

3. angular resolution: for each pair $s$ and $t$ of curves representing two consecutive edges incident on a vertex $v$, the angle between the tangent of $s$ at $v$ and the tangent of $t$ at $v$ should be large. Ideally, we would like the measure of each such angle to be $\Theta(1 / d(v))$, where $d(v)$ denotes the degree of $v$.

^ Partially supported by ONR grant N00014-96-1-0829.

$\star \star$ Also at Max-Planck-Institut für Informatik.

$\star \star \star *$ Partially supported by NSF grant CCR-9732300 and ARO grant DAAH04-96-1-0013. 
Thus, we are interested in a study of methods for drawing planar graphs with smooth edges, small area, and ideal angular resolution. The particular emphasis in this paper is to consider methods for drawing edges with polylines such that each piece of the polyline is drawn with a circular arc. This is a strict generalization of the usual piecewise-linear polylines $[8,10]$, since a straight line segment can be viewed as an arc of a circle of infinite radius. In this paper we address the following questions: What area is achievable for drawings with good angular resolution that use single circle arcs for edges? What area is achievable for drawings that use at most two circular arcs per edge and have good angular resolution? What is the fewest number of circular arcs needed to achieve $O(n) \times O(n)$ area, good angular resolution, and $C^{1}$-continuity for edges?

\subsection{Prior Related Work}

There is a rich body of knowledge that has been developed for drawing planar graphs. Early work by Wagner [14], Fary [4], and Tutte [13] focused on drawings of planar graphs using straight line edges, without much attention paid to other aesthetic or complexity issues. Indeed, the drawings produced using these early techniques can in many cases require exponential area. Later de Fraysseix et al. [3] and then Schnyder [12] showed that one can draw a planar graph with straight line edges and vertices placed at grid points in an $O(n) \times O(n)$ integer grid. Still, the drawings produced from these algorithms have a weakness, which is not as prevalent in the algorithms based on Tutte's approach: namely, the area-efficient straight-line drawings can produce very small angles between consecutive edges incident upon the same vertex (poor angular resolution). In fact, it has been proven [11] that there exist graphs that always require exponential area for straight-line embeddings maintaining good angular resolution.

The problem of drawing planar graphs with good angular resolution was addressed by Formann et al. [5], Garg and Tamassia [6], and Kant [9,10], who showed that one could in fact simultaneously achieve $O(n) \times O(n)$ area and an angular resolution of $\Theta(1 / d(v))$ for each vertex $v$, by drawing a planar graph using piecewise linear polylines with at most three bends each. Gutwenger and Mutzel [8] improved the constant factors for such drawings, establishing that one could draw an $n$-vertex planar graph in a $(2 n-5) \times(3 n / 2-7 / 2)$ grid with at least $2 / d_{\max }$ angular resolution using piecewise linear polylines with at most three bends each, where $d_{\max }$ is the maximum degree of the graph. Goodrich and Wagner [7] showed that one could in fact achieve $O(n) \times O(n)$ area with an angular resolution of $\Theta(1 / d(v))$ for each vertex $v$, using piecewise linear polylines with only two bends each. They also showed that one could achieve the same area and angular resolution bounds using smooth degree-3 (Bézier) curves.

\subsection{Our Results}

In this paper we provide answers to the questions posed above. Specifically, we show the following: 
- There exists an $n$-vertex planar graph $G$ that requires area exponential in $n$ for any drawing of $G$ that uses single circle arcs for edges and has good angular resolution.

- We can draw an $n$-vertex planar graph $G$ in an $O(n) \times O(n)$ grid with angular resolution $\Theta(1 / d(v))$ for each vertex $v$ in $G$ using at most two circular arcs per edge. In fact, in this case we use circular arcs of infinite radius so that the polylines are piecewise linear with at most one bend each, while still maintaining good angular resolution and $O(n) \times O(n)$ area.

- We can draw an $n$-vertex planar graph $G$ in an $O(n) \times O(n)$ grid with angular resolution $\Theta(1 / d(v))$ for each vertex $v$ in $G$ using $C^{1}$-continuous curves that consist of at most three circular arcs.

Our lower-bound proof is based on a non-trivial analysis of a circular-arc drawing of the well-known nested-triangles graph. Our algorithm is based on a careful modification of the incremental approach to planar graph drawing utilized by de Fraysseix et al. [3] similar to the approach used by Goodrich and Wagner [7]. We describe the main ideas behind these results in the sections that follow, beginning our discussion with the algorithm.

\section{Algorithm}

We now describe an efficient algorithm, OneBend, to embed any planar graph on an $O(n) \times O(n)$ grid while maintaining good angular resolution, $\Theta(1 / d(v))$, for each vertex $v$, and using at most one bend per edge. Following the methods of de Fraysseix et al. [3] and Kant [10], we insert vertices sequentially by their canonical ordering, generating subgraphs $G_{1}, G_{2}, \ldots, G_{n}$ in the process. Recall that in the canonical order, vertices are labeled $v_{1}, v_{2}, \ldots, v_{n}$ and graph $G_{i}$ is defined to be the subgraph induced on the vertices $v_{1}, v_{2}, \ldots, v_{i}$. Graph $G_{i}$ is 2 -connected and its external face is a cycle $C_{i}$. Furthermore, in graph $G_{i+1}$, the new vertex, $v_{i+1}$ has all of its neighbors on the external face of $C_{i}$.

In the manner of Goodrich and Wagner [7], we use a box around each vertex of size proportional to its degree but guarantee that each edge drawn contains at most one bend rather than the previous best known method using two. To generate a subgraph $G_{k+1}$ from $G_{k}$ by inserting a vertex $v_{k+1}$ and its associated box, we will need to perform a few operations and maintain a few sets. Let $w_{1}=v_{1}, w_{2}, \ldots, w_{m}=v_{2}$ be the vertices of the exterior face $C_{k}$ of $G_{k}$ in order. For a particular subgraph $G_{k}$ and vertex $v_{k+1}$, we refer to $w_{l}$ and $w_{r}$ as the leftmost and rightmost neighbors of $v_{k+1}$ on $C_{k}$; see Figure 1(a).

\subsection{Vertex Joint Box}

We associate with every vertex $v \in V$ a joint box centered around $v$, rotated $45^{\circ}$, and having width and height $4 d(v)+4$ units, see Figure 1(b). For notational convenience, if $v$ is clear from the context, then we will use $d$ to denote the degree, $d(v)$, of $v$. Thus, if $v$ is located at position $(i, j)$, the four corners of the 


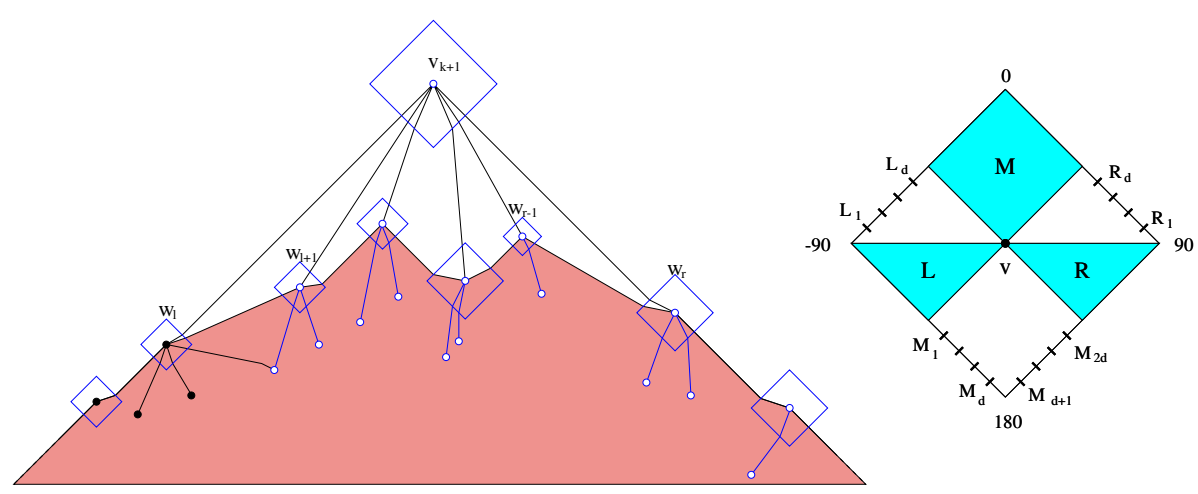

(a)

(b)

Fig. 1. (a) Graph $G_{k+1}$ after inserting $v_{k+1}$. The shaded part is $G_{k}$ and all unfilled vertices are part of the shifting set $M_{k+1}\left(v_{k+1}\right)$. (b) The joint box for a vertex, v.

box are $(i \pm 2 d+2, i)$ and $(i, j \pm 2 d+2)$. We break the box into two types of alternating regions, free regions and port regions. For each free region there is at most one edge passing through it to $v$. Each port region consists of a collection of $d$ ports and every edge inside the port region passes through a unique port. Define the free regions using angular coordinates clockwise around $v$ as follows:

- Free region $M$ lies between $-45^{\circ}$ and $45^{\circ}$.

- Free region $R$ lies between $90^{\circ}$ and $135^{\circ}$

- Free region $L$ lies between $-135^{\circ}$ and $-90^{\circ}$.

In between each of these regions are the port regions. For reference, we label the ports between $L$ and $M$ upward as $L_{1}, \ldots L_{d}$ and similarly between $R$ and $M$. The ports between $L$ and $R$ are labelled $M_{1}, M_{2 d}$ in counterclockwise order.

The algorithm draws each edge in $E$ by "routing" it through a port in the joint box of one of the two vertices. Each edge consists of two connected edge segments. The first edge segment, the port edge segment, connects a vertex with one of its ports while the second segment, the free edge segment, connects the vertex to one of its neighbor's ports.

\subsection{The Invariants}

In order to incrementally construct our embedding, we maintain invariants similar to those of de Fraysseix et al. [3] and Goodrich and Wagner [7] with two important differences (a slight change in invariant three and a new invariant four):

1. The vertices and the ports of the joint boxes have integer coordinates.

2. Let $w_{1}=v_{1}, w_{2}, \ldots, w_{m}=v_{2}$ be the vertices on the exterior face $C_{k}$ of $G_{k}$ in order. Then $x\left(w_{1}\right)<x\left(w_{2}\right)<\cdots<x\left(w_{m}\right)$, where $x\left(w_{i}\right)$ is $w_{i}$ 's $x$-coordinate. 
3. Edge $\left(w_{i}, w_{i+1}\right)$, for $0<i<m$ has the free edge segment with slope \pm 1 .

4. For every vertex $v$ there is at most one edge segment crossing each of its free regions. All other edge segments are port edge segments.

Notice that if invariant four holds for the embedding $G_{k}$, by the definition of the joint box and location of the port regions, $G_{k}$ has angular resolution no worse than $\Theta(1 / d(v))$, for each vertex $v$.

\subsection{The Shifting Set}

During each insertion, we must create space for the vertex joint box to "see" its leftmost and rightmost neighbors without the box touching any of the neighbors along the face in between. To do this, we need to shift the vertices along the external face by a certain amount. However, in order for the invariants and planarity to be guaranteed other vertices must also be shifted at the same time. As in de Fraysseix et al. [3] and Goodrich and Wagner [7], we define the shifting set for a vertex $w_{i}$ on the external face of $G_{k}$ as $M_{k}\left(w_{i}\right)$. For any graph $G_{k}$, we define $M_{k}\left(w_{i}\right) \subseteq V$ so that the following conditions hold:

1. $w_{j} \in M_{k}\left(w_{i}\right)$ if and only if $j \geq i$.

2. $M_{k}\left(w_{1}\right) \supset M_{k}\left(w_{2}\right) \supset \cdots \supset M_{k}\left(w_{m}\right)$.

3. For any nonnegative numbers $\delta_{1}, \delta_{2}, \ldots, \delta_{m}$, if we sequentially translate all vertices in $M_{k}\left(w_{i}\right)$ with distance $\delta_{i}$ to the right $(i=1,2, \ldots, m)$, then the embedding of $G_{k}$ remains planar. ${ }^{1}$

Recall that for a vertex $v=v_{k+1}, w_{l}$ and $w_{r}$ are the leftmost and rightmost neighbors of $v$ on $C_{k}$. Starting with the initial shifting set at $k=3$, we construct $M_{k+1}\left(w_{i}\right)$ recursively as follows: $M_{k+1}\left(w_{i}\right)=M_{k}\left(w_{i}\right) \cup v_{k+1}, M_{k+1}\left(v_{k+1}\right)=$ $M_{k}\left(w_{l+1}\right) \cup v_{k+1}, M_{k+1}\left(w_{j}\right)=M_{k}\left(w_{j}\right)$, for $i \leq l$ and $j \geq r$.

This construction allows us to guarantee that the above three conditions of the shifting sets are maintained. Intuitively, after a vertex $w_{i}$ is removed from the external face by another vertex $v_{k+1}$, it always shifts exactly with $v_{k+1}$. During any shift, vertices can only get farther apart in the $x$-direction. Note that in our algorithm, when a vertex is shifted, its joint box is also shifted, i.e., the ports move as well.

\subsection{The Construction}

We now show how algorithm OneBend iteratively constructs graphs $G_{1}, G_{2}, \ldots$, $G_{n}$. It is trivial to construct the initial cases of $G_{1}, G_{2}$, and $G_{3}$, i.e. inserting the first three vertices. Suppose we have embedded $G_{k}$ with exterior face $C_{k}$. Let $C_{k}=\left(v_{1}=w_{1}, w_{2}, \ldots, w_{m}=v_{2}\right)$ be the exterior face of $G_{k}$. To construct $G_{k+1}$, let $v=v_{k+1}$ be the next vertex in the canonical ordering and recall that $w_{l}$ and $w_{r}$ are, respectively, the leftmost and rightmost neighbors of $v$ on the face $C_{k}$.

\footnotetext{
${ }^{1}$ Note that many vertices will move several times; e.g. all points in $M_{k}\left(w_{i}\right) \backslash M_{k}\left(w_{i+1}\right)$ will be translated by $\delta_{1}+\delta_{2}+\cdots+\delta_{i}$.
} 
Let $d, d_{l}, d_{r}$ be the respective degrees of $v, w_{l}$, and $w_{r}$. Let $p_{l}$ be the first unused $R_{i}$ port in $w_{l}$ 's joint box. Similarly, let $p_{r}$ be the first unused $L_{i}$ port in $w_{r}$ 's joint box. Recall since each port region has at least $d$ ports available there is always an unused port.

We insert $v$ by shifting all vertices in the shifting set $M_{k}\left(w_{l+1}\right)$ by $2 d+2$ positions to the right. Additionally we shift all vertices in $M_{k}\left(w_{r}\right)$ by an additional $2 d+2$ positions to the right. This implies all vertices in $M_{k}\left(w_{r}\right)$ actually move $4 d+4$ positions. Finally, we place $v$ at the intersection of lines $l$ and $r$ where $l$ (respectively $r$ ) is the line through $p_{l}$ (respectively $p_{r}$ ) with slope +1 (respectively -1). We route the edges between $v$ and $w_{l}$ through $p_{l}$ and do the same for $w_{r}$. To maintain invariants one and three, notice that if the intersection point has integer coordinates these two invariants hold. Otherwise, by shifting $M_{k}\left(w_{r}\right)$ one additional unit, the intersection point has integer coordinates.

To complete the insertion and the algorithm, we need to draw the edges between $v$ and $w_{i}$, where $l<i<r$. Let $w_{j}$ be the rightmost vertex with an $x$ coordinate less than $v$. We route the edges from $v$ to vertices $w_{i}$, where $l<i \leq j$ through consecutive increasing ports from $M_{1}$ in $v$ 's joint box. Similarly, we route the edges from $v$ to vertices $w_{i}$, where $r>i>j$ through consecutive decreasing ports from $M_{2 d}$ in $v$ 's joint box.

Lemma 1. After shifting, free edge segments remain in their free regions.

Proof Sketch: Consider the free edge segments in the $M$ regions. Notice that these segments are created by a vertex $v$ dominating another vertex $w$. In this case, $w$ joins $v$ 's shifting set and is only shifted when $v$ is shifted. Therefore, the slope remains constant and the free edge segment remains within $M$.

Consider the case when the free edge segment lies in the $L$ region. This implies that the slope of the line is between 0 and +1 . Since shifting only moves vertices farther apart in the $x$-direction, the slope can only get closer to 0 while still remaining in $L$. The argument is similar for $R$.

Lemma 2. After insertion, every free edge segment passes through a free region which contains no other segment.

Proof Sketch: After inserting a vertex $v_{k+1}$, there are two types of edges added: edges between $v_{k+1}$ and the outside neighbors, $w_{l}$ and $w_{r}$, and edges between $v_{k+1}$ and $w_{i}$ where $l<i<r$. In both cases the edge is routed through a port creating one free edge segment and one port edge segment. By construction, a free edge segment of the first type has slope either +1 or -1 and so it lies inside $v_{k+1}$ 's joint box free region $L$ or $R$, respectively. Since $v_{k+1}$ is a new vertex, there are no other segments inside these two free regions.

A free edge segment of the second type intersects the $M$ region of $w_{i}$ 's joint box. Since this can happen at most once, as the vertex is now no longer on an external face, there can be no other free edge segment inside this free region.

Lemma 3. If invariants 1-4 hold for $G_{k}$, then they also hold for $G_{k+1}$. 
Proof Sketch: By the nature of the shifting set, invariants one and two hold (see [7]). Since shifting a vertex involves shifting the entire joint box simultaneously, after every shift operation all port edge segments have unchanged slope. Also, after the two shifting operations, all free edge segments on the exterior face have unchanged, albeit \pm 1 slope, except possibly the free edge segments $\left(w_{l}, w_{l+1}\right)$ and $\left(w_{r-1}, w_{r}\right)$. However, after insertion, these free edge segments are no longer on the exterior face and are instead replaced by two free edge segments between $\left(w_{l}, v\right)$ and $\left(v, w_{r}\right)$ with slope \pm 1 . Therefore, invariant three holds.

By lemmas 1 and 2 and the fact that port edge segments never change slope, we see that invariant four also holds since all edges routed in algorithm OneBend created a port segment and a free edge segment.

Theorem 1. Given a planar graph $G$, algorithm OneBend produces in $O(n)$ time a planar embedding on the $30 n \times 15 n$ grid with angular resolution $\Theta(1 / d(v))$ and using any of the following types of edges: polylines with one bend, or two circular arcs with $C^{0}$-continuity and one knot, or three circular arcs with $C^{1}$-continuity.

Proof Sketch: The original algorithm as stated produces polylines with one bend per edge. This by definition can also be represented by two circular arcs, straight lines, which have a discontinuity at the bend, or knot. Since the points are embedded on the grid, the bends may also be replaced by circular arcs of a relatively small size to ensure $C^{1}$-continuity as well.

It has been shown by Chrobak and Payne [2] how to implement the algorithm of De Fraysseix, et al. [3] in linear time. Their approach can be easily extended to our algorithm. It remains to show that the drawings produced by algorithm OneBend fit on the $30 n \times 15 n$ grid. Every time we insert a vertex $v_{k}$, we increase the grid size by $4 d\left(v_{k}\right)+5$ units. Summing over all the degrees of the vertices we get $\sum_{v \in V} 4 d(v)+5=4(6 n-12)+5 n<30 n$. The final drawing fits inside an isosceles triangle with sides of slope $0,+1,-1$. The width of the base is $30 n$ and so the height is less than $15 n$.

\section{Drawing with Circular Arcs}

Malitz and Papakostas [11] showed that some planar graphs, drawn with straight lines in the $O(n) \times O(n)$ grid must have small angles. More specifically, they found a class of planar graphs, $\mathcal{H}$, whose straight-line planar drawings require exponential area if the angular resolution is good. Suppose we relax the condition that each edge in a graph be drawn with a straight line segment so that each edge is drawn with a circular arc (where a straight line segment is considered an arc from a circle of radius infinity). Can we draw the graphs in $\mathcal{H}$ with angular resolution $\alpha>0$ in an $O(n) \times O(n)$ grid? Surprisingly, as long as $\alpha$ is a constant, the answer is no.

Let $\mathcal{H}=\left\{H_{n}, n \geq 1\right\}$ and $H_{1}$ be a cycle on 3 vertices $P_{1}, Q_{1}$ and $R_{1}$. For $n \geq 2$, the graph $H_{n}$ is constructed from $H_{n-1}$ by adding a cycle on three new vertices $P_{n}, Q_{n}, R_{n}$, and edges $\left(P_{n}, P_{n-1}\right),\left(Q_{n}, Q_{n-1}\right),\left(R_{n}, R_{n-1}\right)$ and 

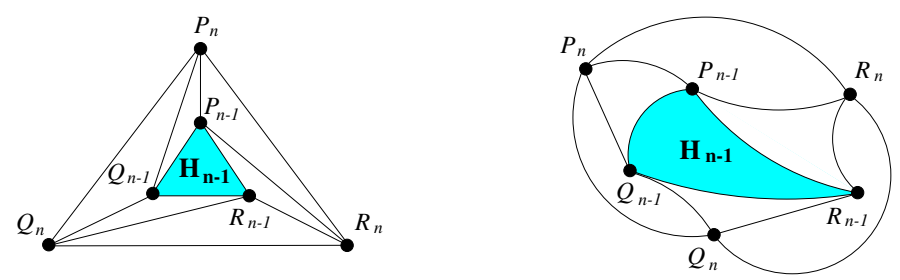

Fig. 2. Graph $H_{n}$ is constructed from $H_{n-1}$ by adding vertices $P_{n}, Q_{n}, R_{n}$ and the edges shown above. The figure on the right shows $H_{n}$ drawn with circular arcs.

$\left(P_{n}, Q_{n-1}\right),\left(Q_{n}, R_{n-1}\right),\left(R_{n}, P_{n-1}\right)$, as shown in Figure 2 . It is easy to check that the graph is planar, triconnected and thus, has a unique embedding. We show that for any planar, circular-arc drawing of $H_{n}$ with angular resolution $\alpha>0$, there exists a constant $c_{\alpha}>1$ such that the area of the drawing is $\Omega\left(c_{\alpha}^{n}\right)$.

Let $\Gamma_{n}$ be a planar circular-arc drawing of $H_{n}$ with angular resolution $0<$ $\alpha \leq \pi / 3$. If $(u, v)$ is an edge in $H_{n}$ then we shall refer to the arc that represents $(u, v)$ in $\Gamma_{n}$ as $\widehat{u v}$, and the line segment that connects $u$ and $v$ as $\overline{u v}$. (Sometimes $u$ or $v$ may not be a vertex of $H_{n}$ but a point on some arc of $\Gamma_{n}$. In this case, $\widehat{u v}$ refers to the portion of the arc that starts at $u$ and ends at $v$.) If $\mathcal{S}$ is a set of arcs in $\Gamma_{n}$ that bounds a region, then we let $\operatorname{Area}(\mathcal{S})$ be its area.

Define regions $\mathcal{S}_{1}, \mathcal{S}_{2}$ and $\mathcal{S}_{3}$ as follows: $\mathcal{S}_{1}=\left\{P_{n-1} \widehat{Q_{n-1}}, \widehat{Q_{n-1}} P_{n}, \widehat{P_{n} \widehat{P_{n-1}}}\right\}$, $\mathcal{S}_{2}=\left\{Q_{n-1} \widehat{R}_{n-1}, R_{n-1} Q_{n}, Q_{n} \widehat{Q}_{n-1}\right\}$ and $\mathcal{S}_{3}=\left\{R_{n-1} \widehat{P}_{n-1}, P_{n-1} R_{n}, R_{n} \widehat{R_{n-1}}\right\}$. We shall show in the next two lemmas that the region enclosed by the three arcs in $\mathcal{S}_{1}$ cannot be arbitrarily small. If all the arcs in $H_{n}$ are straight lines, this fact is easy to prove. However, for circular-arc drawings, we need to take into account that the arcs can have different curvatures. Nonetheless, the requirement that the tangents of two incident arcs must form at least an angle $\alpha>0$ will allow us to show that, regardless of the curvatures of the three $\operatorname{arcs}$ in $\mathcal{S}_{1}, \operatorname{Area}\left(\mathcal{S}_{1}\right)$ is proportional to $\left|\overline{P_{n-1} Q_{n-1}}\right|^{2}$ as well as the area enclosed by $P_{n-1} \widehat{Q}_{n-1}$ and $\overline{P_{n-1} Q_{n-1}}$. Similarly, the areas of the regions enclosed by the arcs in $\mathcal{S}_{2}$ and $\mathcal{S}_{3}$ cannot be arbitrarily small.

Let $Z_{n-1}$ be the midpoint of $P_{n-1} \widehat{1}_{n-1}$. Consider the two circular arcs that pass through $P_{n-1}$ and $Z_{n-1}$ such that the tangents of the arcs form an angle $\alpha$ with $P_{n-1} \widehat{Q}_{n-1}$. Let $\widehat{a}$ be the arc that lies on the outside face of $H_{n-1}$. Let $\widehat{b}$ be the corresponding arc that passes through $Q_{n-1}$ and $Z_{n-1}$, see Figure 3 .

$\operatorname{Lemma}$ 4. $\operatorname{Area}\left(\mathcal{S}_{1}\right) \geq \operatorname{Area}\left(\left\{\widehat{a}, P_{n-1} \widehat{1}_{n-1}\right\}\right)$.

Proof: Let $l$ be the perpendicular bisector of $\overline{P_{n-1} Q_{n-1}}$. Without loss of generality, assume that $P_{n}$ lies on $l$ or on the same side of $l$ as $Q_{n-1}$. Notice that if $P_{n} \neq Z_{n-1}, \widehat{P_{n-1}} P_{n}$ is always above $\widehat{a}$ except at its endpoint, $P_{n-1}$. Otherwise, the angular resolution of $\Gamma_{n}$ is violated or $P_{n}$ lies below $\widehat{a}$ and hence on the wrong side of $l$. Furthermore, $\widehat{Q_{n-1}} P_{n}$ cannot intersect $\widehat{a}$, except possibly at $Z_{n-1}$. If it does, it crosses $l$ and has to intersect $\widehat{P_{n-1} P_{n}}$ as well, contradicting the assumption that $\Gamma_{n}$ is a planar drawing. Thus, both $\widehat{P_{n-1} P_{n}}$ and $\widehat{Q_{n-1}} P_{n}$ 

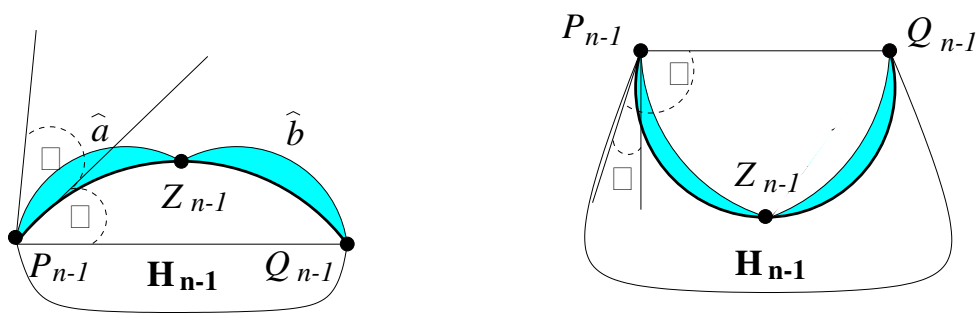

Fig. 3. Arcs $\widehat{a}$ and $\widehat{b}$ pass through $P_{n-1}, Z_{n-1}$ and $Q_{n-1}, Z_{n-1}$ respectively. Their tangents form an angle $\alpha$ with the tangents of $P_{n-1} Z_{n-1}$ and $Q_{n-1} Z_{n-1}$. The shape of the region bounded by $\widehat{a}$ and $P_{n-1} \widehat{Z}_{n-1}$ depends on the concavity/convexity of $P_{n-1} Q_{n-1}$ and $\alpha$.

do not cross $\widehat{a}$; $\widehat{a}$ must lie in the region enclosed by $\mathcal{S}_{1}$. By symmetry, if $P_{n}$ lies on the same side of $l$ as $P_{n-1}$, then $\widehat{b}$ must lie in the region enclosed by $\mathcal{S}_{1}$.

Lemma 5. There exists positive constants $k_{\alpha}$ and $k_{\alpha}^{\prime}$ such that

i. $\operatorname{Area}\left(\left\{\widehat{a}, P_{n-1} \widehat{Z}_{n-1}\right\}\right) \geq k_{\alpha} \mid{\overline{P_{n-1} Q_{n-1}}}^{2}$ and

ii. $\operatorname{Area}\left(\left\{\widehat{a}, P_{n-1} \widehat{Z}_{n-1}\right\}\right) \geq k_{\alpha}^{\prime} \operatorname{Area}\left(\left\{P_{n-1} \widehat{Q}_{n-1},{\overline{P_{n-1} Q_{n-1}}}\right\}\right)$.

The proof of the above lemma is omitted from this extended abstract.

Note that $k_{\alpha}$ and $k_{\alpha}^{\prime}$ are not dependent on $\gamma$ and hence the result can be extended to the other arcs, $Q_{n-1} \widehat{R}_{n-1}$ and $R_{n-1} \widehat{P}_{n-1}$.

Theorem 2. Any planar, circular-arc drawing of $H_{n}$ that has constant angular resolution $\alpha>0$ has area $\Omega\left(c_{\alpha}^{n}\right)$ where $c_{\alpha}>1$.

Proof: Let $\Gamma_{n}^{*}$ be a planar, circular-arc drawing of $H_{n}$ with minimum area $A_{n}$. Let $B_{n-1}$ denote the area occupied by $H_{n-1}$ in $\Gamma_{n}^{*}$. Clearly, $B_{n-1} \geq A_{n-1}$. Then,

$$
\begin{aligned}
A_{n} \geq & B_{n-1}+\operatorname{Area}\left(\mathcal{S}_{1}\right)+\operatorname{Area}\left(\mathcal{S}_{2}\right)+\operatorname{Area}\left(\mathcal{S}_{3}\right) \\
\geq & B_{n-1}+\frac{1}{2}\left[k_{\alpha}\left|\overline{P_{n-1} Q_{n-1}}\right|^{2}+k_{\alpha}^{\prime} \operatorname{Area}\left(\left\{\bar{P}_{n-1} \widehat{Q}_{n-1}, \overline{P_{n-1} Q_{n-1}}\right\}\right)+\right. \\
& k_{\alpha}\left|\overline{Q_{n-1} R_{n-1}}\right|^{2}+k_{\alpha}^{\prime} \operatorname{Area}\left(\left\{Q_{n-1} \widehat{R}_{n-1}, \overline{Q_{n-1} R_{n-1}}\right\}\right)+ \\
& \left.k_{\alpha}\left|\overline{R_{n-1} P_{n-1}}\right|^{2}+k_{\alpha}^{\prime} \operatorname{Area}\left(\left\{R_{n-1} \widehat{P}_{n-1}, \overline{R_{n-1} P_{n-1}}\right\}\right)\right] \\
\geq & B_{n-1}+\frac{\min \left(k_{\alpha}, k_{\alpha}^{\prime}\right)}{2}\left[\left|\overline{P_{n-1} Q_{n-1}}\right|^{2}+\left|\overline{Q_{n-1} R_{n-1}}\right|^{2}+\left|\overline{R_{n-1} P_{n-1}}\right|^{2}\right. \\
& +\operatorname{Area}\left(\left\{P_{n-1} \widehat{Q}_{n-1}, \overline{P_{n-1} Q_{n-1}}\right\}\right)+\operatorname{Area}\left(\left\{Q_{n-1} \widehat{R}_{n-1}, \overline{Q_{n-1} R_{n-1}}\right\}\right) \\
& \left.+\operatorname{Area}\left(\left\{\widehat{R}_{n-1} \widehat{P}_{n-1}, \overline{R_{n-1} P_{n-1}}\right\}\right)\right] \\
\geq & B_{n-1}+\frac{\min \left(k_{\alpha}, k_{\alpha}^{\prime}\right)}{2}\left[\left(\operatorname{Area}\left(\left\{\overline{P_{n-1} Q_{n-1}}, \overline{Q_{n-1} R_{n-1}}, \overline{R_{n-1} P_{n-1}}\right\}\right)+\right.\right. \\
& \operatorname{Area}\left(\left\{P_{n-1} \widehat{Q}_{n-1}, \overline{P_{n-1} Q_{n-1}}\right\}\right)+\operatorname{Area}\left(\left\{Q_{n-1} \widehat{R}_{n-1}, \overline{Q_{n-1} R_{n-1}}\right\}\right)+ \\
& \text { Area } \left.\left(\left\{\widehat{R}_{n-1} \widehat{P}_{n-1}, \overline{R_{n-1} P_{n-1}}\right\}\right)\right] \\
\geq & B_{n-1}+\frac{\min \left(k_{\alpha}, k_{\alpha}^{\prime}\right)}{2} B_{n-1} \geq\left(1+\frac{\min \left(k_{\alpha}, k_{\alpha}^{\prime}\right)}{2}\right) A_{n-1} \cdot
\end{aligned}
$$


Note that (1) follows from lemmas 4 and 5 and (2) from the fact that $B_{n-1} \geq$ $\operatorname{Area}\left(\left\{\overline{P_{n-1} Q_{n-1}}, \overline{Q_{n-1} R_{n-1}}, \overline{R_{n-1} P_{n-1}}\right\}\right)+\operatorname{Area}\left(\left\{P_{n-1} \widehat{Q}_{n-1},{\overline{P_{n-1} Q_{n-1}}}\right\}\right)+$

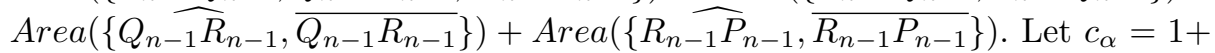
$\min \left(k_{\alpha}, k_{\alpha}^{\prime}\right)$. Since $A_{1}$ is at least some constant $a_{1}>0$, by induction, $A_{n} \geq$ $c_{\alpha}^{n-1} a_{1}$.

\section{Acknowledgements}

We would like to thank Anhua Lin for helpful discussions and suggestions.

\section{References}

1. G. Di Battista, P. Eades, R. Tamassia, and I. Tollis. Graph Drawing: Algorithms for the Visualization of Graphs. Prentice Hall, Englewood Cliffs, NJ, 1999.

2. M. Chrobak and T. Payne. A linear-time algorithm for drawing planar graphs. Inform. Process. Lett., 54:241-246, 1995.

3. H. de Fraysseix, J. Pach, and R. Pollack. How to draw a planar graph on a grid. Combinatorica, 10(1):41-51, 1990.

4. I. Fary. On straight lines representation of planar graphs. Acta Sci. Math. Szeged, 11:229-233, 1948.

5. M. Formann, T. Hagerup, J. Haralambides, M. Kaufmann, F. T. Leighton, A. Simvonis, Emo Welzl, and G. Woeginger. Drawing graphs in the plane with high resolution. SIAM J. Comput., 22:1035-1052, 1993.

6. A. Garg and R. Tamassia. Planar drawings and angular resolution: Algorithms and bounds. In Proc. 2nd Annu. European Sympos. Algorithms, volume 855 of Lecture Notes Comput. Sci., pages 12-23. Springer-Verlag, 1994.

7. M. T. Goodrich and C. G. Wagner. A framework for drawing planar graphs with curves and polylines. In Graph Drawing '98, pages 153-166, 1998.

8. C. Gutwenger and P. Mutzel. Planar polyline drawings with good angular resolution. In Graph Drawing '98, pages 167-182, 1998.

9. G. Kant. Drawing planar graphs using the lmc-ordering. In Proc. 33th Annu. IEEE Sympos. Found. Comput. Sci., pages 101-110, 1992.

10. G. Kant. Drawing planar graphs using the canonical ordering. Algorithmica, 16:4-32, 1996. (special issue on Graph Drawing, edited by G. Di Battista and R. Tamassia).

11. S. Malitz and A. Papakostas. On the angular resolution of planar graphs. SIAM J. Discrete Math., 7:172-183, 1994.

12. W. Schnyder. Embedding planar graphs on the grid. In Proc. 1st ACM-SIAM Sympos. Discrete Algorithms, pages 138-148, 1990.

13. W. T. Tutte. How to draw a graph. Proceedings London Mathematical Society, 13(52):743-768, 1963.

14. K. Wagner. Bemerkungen zum vierfarbenproblem. Jahresbericht der Deutschen Mathematiker-Vereinigung, 46:26-32, 1936. 